great relief and cure the patient before his bladder is diseased. The dangers are almost nil because the great source of sepsis, the fetid urine, can not touch the wound if it is well packed with iodoform gauze until it heals from the bottom. Open the perineum by the bilateral section, proceed as though you intended to enucleate the prostate, but don't. Dress as above described, and the results will prove satisfactory in most instances.

Hal. C. Wyman.

\section{Medical Practice in Colorado.}

Denver, Col., July 24, 1893.

To the Editor:-I should be glad to have you publish the following regarding our present Colorado Iaw :

Hereafter the Colorado State Board of Medical Examiners will recognize only diplomas from three year schools as entitling their holders to license. The courses of lectures must have been of at least twenty weeks each, and given in three separate years, and a preliminary examination must have been required. Instruction must have been given in anatomy, chemistry, physiology, pathology, materia medica and therapeutics, obstetrics and gynecology, surgery, medical jurisprudence, theory, and practice of medicine and hygiene.

In default of such a diploma the candidate for license must pass an examination in anatomy, chemistrv, physiology, pathology, surgery, obstetrics and gynecology, and theory and practice of medicine. Yours very truly, J. N. HALL, M.D., Secretary.

\section{An Inquiry.}

FulTos, N. Y., July 24, 1893.

To the Editor:-Will some of your contributors give reasons why venesection is not as useful in relieving acute diseases, viz: pneumonia, now, as it was fifty years ago? Also the causes why epistaxis and other hemorrhages in the young, are so uncommon now compared with 1840 to 1870 ? During that period I was frequently applied to, to arrest nose bleed, more often for the young under 20 years of age. For the last twenty years, I do not remember of being called in a single instance excepting in a few cases of severe exanthematic to relieve nose bleeding. Has the diet, the mode of living, the less physical labor endured, or what has made the difference?

C. G. BACON.

There are no Classes in the Association-All are Equal and Pay Annually.

To the Editor:-Please tell $\mathrm{me}$ how long must a paying member continue his dues until he is a life member?

Sparks, Neb., July 26, 1893.

L. K. W. S.

\section{BOOK NOTICES.}

System of Diseases of the Ear, Nose and Throat. Edited by Charles H. Burnetr, A.M., M.D., Emeritus Professor of Otology in the Philadelphia Polyclinic, etc. Vol. I. Illustrated. Philadelphia: J. B. Lippincott Company. 1893. Pp. 800. Price per vol. in eloth, $\$ 6$.

Dr. Burnett has produced a valuable and practical book. It consists of monographs upon the various subdivisions of these subjects by twenty-three writers. They aim to give practical conclusions arrived at by other investigators and by themselves aside from theories.

There are two volumes. The first is devoted to the ear, nose and naso-pharynx; and the second to diseases of the pharynx and larynx. The purpose has been to make this work a complete, exhaustive and authoritative treatise with special reference to diagnosis and treatment.

Dr. H. Richards describes the instruments and methods of examination of patients with diseases of the ear. Too much space is given to the old, to the exclusion of the new and important instruments and methods. Fig. 14 illustrates "Politzer's method of inflation of tympana." It represents the forcing of a column of air upward in the direction of the nasal duct, instead of horizontally backwards, as it ought to be, in a line with the orifice of the Eustachian tube. It is plain that a jet of air, like a jet of any gas, or water. should be propelled in the direction of the tube through which it is intended to pass, and such an error as is perpetuated here should be eliminated from a work of this eharacter. Fig. 18 illustrates catheterization. The Politzer air bag is fitted directly into the catheter, without any intervening soft rubber tubing, as it is done in Vienna. Many American aural surgeons have used for years an improvement on this method that saves the patient much unnecessary suffering. It consists of interposing a soft rubber tube between the air bag and the catheter tip. By this means the pain-producing, jerking motion imparted to the catheter with each compression of the bag is prevented. These improvements are important and should be recorded, especially those made by our own countrymen, and they are many. The great strides made in improvements in compressed air apparatus and instruments as employed in Berlin, New York and Chicago are not mentioned in this paper. Dr. G. Bacon's paper on acute inflammation of the middle ear is full of excellent advice and good sense. He is to be commended for condemning the universal practice of taking large doses of quinine for "colds in the head," especially when the ear is involved. He is one of the very few writers who properly pleuralize the Latin name for drumheads (membrane tympanorum). He sensibly discountenances the use of the Valsalvan and Politzer methods by patients. They usually do themselves more harm than good. His paper is illustrated by seven colored drawings.

Dr. S. Sexton writes on chronic catarrh of the middle ear. He treats the subject with special reference to the removal of two larger ossicles. The operation is described and strongly recommended. Nothing is said of the deplorable results that sometimes follow this operation-suppuration, vertigo, apparently intensified tinnitus, reproduction of the drumhead and total deafness. Both sides of this question have been fairly and impartially presented in the meetings of the otological section of the American Medical Association, and one naturally looks for the same here.

Dr. C. J. Colles has an exceedingly valuable contribution on the middle ear affections due to the various dyscrasias. It evidences painstaking and exhaustive research.

Dr. R. Barclay gives the best description of fungoid growths of the ear that we have ever seen. There are twenty-five pages, well illustrated, on this subject alone.

Dr. C. H. Burnett's paper on chronic suppuration of the middle ear is a thoroughly practical one. He describes minutely the operation for incision of the hammer and anvil, and is in accord with the best authorities in advising this procedure in the very intractable cases. His statement that peroxide (dioxide) of hydrogen is decomposed by warming it before putting it in the ear is a mistake. At the temperature of the blood the efficacy of a good $\mathrm{H}_{2} \mathrm{O}_{2}$ is retained, and it is conducive to the comfort of the patient. While he does not endorse Stacke's operation for opening the attic and antrum and excision of the ossicles, he gives a brief but clear account of it. This procedure is about to be tried by Toeplitz and others of New York, and it has been dore recently in Chicago, with successful results, by the aural surgeons of the Illinois Charitable Eye and Ear inflrmary. It is one of the most difficult and dangerous operations in surgery.

In Part II, Dr. A. W. MacCoy has a beautifully illustrated article on the anatomy and physiology of the nose and pharynx.

Dr. C. Wagner's paper on the treatment of the nose and throat is of great practical value to the general practitioner. Yet it is regrettable that he endorses the use of the abominable nasal douche, In recommending the use of pyoktannin (which kind?) in sprays and powder. he omits the necessary warning regarding its intense staining propertyyellow or violet.

Iaryngologists will be surprised to find in the first line of Dr. Bosworth's paper on acute rhinitis the word he has so uncompromisingly condemned-catarrhal. He has re- 